\title{
Very High-Performance Echo Canceller for Digital Terrestrial Television in Single Frequency Network
}

\author{
El Miloud Ar-Reyouchi ${ }^{1}$ \\ Telecommunications Computer \\ Science, Abdelmalek Essaadi \\ University and SNRT \\ Tetouan, Rabat, Morocco
}

\author{
Yousra Lamrani $^{2}$ \\ Kamal Ghoumid ${ }^{3}$ \\ Electronic informatics \\ Telecommunications, ENSAO \\ Mohammed I University \\ Oujda, Morocco
}

\author{
Salma Rattal ${ }^{4}$ \\ Electrical engineering \\ FSTM Hassan II University \\ Casablanca, Morocco
}

\begin{abstract}
The principal aim of this paper is to cancel out the natural and man-made echoes in single-frequency networks (SFN). The challenge is to detect and remove feedback echoes and enhance the intelligibility of the essential parameters in SFN of digital terrestrial television broadcasting (DTTB) transmitter systems, especially the Modulation Error Ratio (MER), with optimizing coverage areas. We suggest a Digital Video Broadcasting (DVB) gap filler (GF) with two types of echo cancelling: Digital Adaptive Equalizer (DAE) and Doppler Enhanced Echo Canceller (DEEC). The proposed GF outperforms standard GF (SGF), finite impulse response filter (FIR GF), and adaptive GF (AGF) techniques by $33 \%, 17 \%$, and $13 \%$, respectively. Furthermore, the obtained MER makes the proposed GF (PGF) ideal for operating in SFN using Coded Orthogonal Frequency Division Multiplex (COFDM) technique.
\end{abstract}

Keywords-Gap filler; Digital Adaptive Equalizer (DAE); Doppler Enhanced Echo Canceller (DEEC); Single-Frequency Networks (SFN); Coded Orthogonal Frequency Division Multiplex (COFDM)

\section{INTRODUCTION}

In DTTB, the terrestrial digital TV, and gap-filler transmitters broadcast the same TV service using the single frequency. The Coded Orthogonal Frequency Division Multiplex (COFDM) techniques [1],[2] allow TV transmitters to create an SFN, which is widely used for economizing frequency [3]. The presence of a GI (guard interval) [4], in COFDM, gives it excellent robustness to lute against echoes derived from multipath interference. The coincidence of several TV broadcasts produces different types of echoes, whether artificial or natural. The echoes may appear when receiving simultaneously [5] the same signal from several transmitters. Another reason that can cause echoes is the reflection of the signal on large objects such as mountains.

To improve wide-area performance applying SFN synchronization, the studies in [6],[7] have treated various network architectures depending on different parameters like COFDM technology, the selected scattering power, and a perfect adaptation measurement[8] with a suitable antenna[9] for the reception of DVB.

In SFN, the practical network planning approach of the transmitter's location and antenna orientation are always necessary to reduce the interference. Echoes can also be reduced by decreasing the number of transmitters per local area. The selection of a transmitter with multiple antennas to the transmission and reception MIMO (multiple inputs, multiple outputs) [10], [11] heights they can promote smooth operation of the self-interference cancellation technique.

In the traditional Multi-Frequency Networks (MFN) technology, each transmitter uses a different frequency to avoid undue interference between its neighbors. Interference can be assimilated to an echo [12] if the content of the signal is the same on both transmitters.

In SFN, the echoes and interferences are avoided by COFDM modulation on which they are based. Since GF transmits and receives on different frequencies, it can also operate on the identical frequency with low-medium power DVB transmitters. This research approach is chosen to provide additional converge on those blind spots not covered by the high power transmitter using efficient proposed GF (PGF) operating in SFN using the same channel. The strength of the PGF is reflected in the ability to annul the feedback echoes effectively, increasing the frequency efficiency. The PGF is based on two echo-canceling DAE and DEEC. The DEEC is based on the least-mean-square (LMS) adaptive filter [13] using the LMS algorithm [14], the DEEC provides a filter that models the channel between the transmitter (Tx) and receiver (Rx) antennas. With DAE [15], the performance of a DTTB for Quadrature amplitude modulation (QAM) can be ameliorated considerably. Many studies have explored different aspects of the behavioral feedback path, such as [16]. In [17], the authors analyze the required overhead to ensure a target average signal-to-interference-plus-noise ratio (SINR) using Time-division multiple access (TDMA) of each node. In [18], the interference cancellation (IC) reduces interference effect, and the overlap ratio could be enhanced while maintaining a high quality of service (QoS). The echo cancellation method has been studied in [19],[20], and analyzed in a deferent domain such as in [21] for voice over IP (VoIP) communication acoustic echo.

In this work, broadcast technology is adopted (one point to all other points, the authors present two echo cancellation methods as a critical technique to solve the echo and interference problem in the SFN environment using DAE and DEEC like an echo cancellation method. Furthermore, the ideal proposed technique should be compared with other research re- 
sults, such as [22]. This paper does not only ensure the safe and robust operation of the repeater against the dynamics of the environment [23] but also can suppress Doppler Effect and avoid the interferences and echoes to the received signal from the transmitter in its overall coverage area. Overall, it has to be noted that the applied Echo cancellation [24] techniques not only help to improve isolation [25] but also gives perfect emission-reception isolation. Our goal is to get satisfaction levels of $80-85 \%$ of a parameter, which have a direct relationship with the echoes suppression, especially the MER. The MER is a paramount measure, and DVB widely requires it for OFDM signal performance in SFN, it is analyzed in [26] as received signal power in an actual digital television transmitter. MER is affected by the delay of received signals [27] from the SFN network, and the result of MER showed the quality of service (QoS) at the receiver.

The remaining of this paper is classified as follows. In the second section, we briefly introduce a general system Performance. The third section exposes and discusses the problem with the remedy. The fourth section presents and discovers the echo canceller utility and service, such as DAE \& DEEC. The fifth section deals with the summary of the experimental results obtained. Finally, the sixth section presents conclusions.

\section{General PERFormance}

\section{A. General Performance}

The transmission path is known as "Rician channel," which takes into consideration the effect of multipath signals, noise, and the dominating direct signal path between Tx and Rx.

Temporal delay shall be estimated according to the following formula: $\lambda=c / f=c T \Rightarrow \Delta d=c \tau$ where $\lambda$ is the wavelength, $c$ is the speed of light, and $f$ is frequency. The offset delay is more important for remote transmitters $(200 \mu$ s for 60 $\mathrm{km})$. Consequently, at the receiver side, due to the presence of the multiple electromagnetic paths, more than one signal is received by a specific instance, and each one of them reaches at different times $\tau(t)$ with different energy strengths $\beta(t)$ and different angles $\alpha(t)$.

Let $S(t)$ be the transmitted complex signal having a carrier frequency $f_{0}$ modulated by a baseband complex signal $x(t)$, and it can be written as:

$S(\mathrm{t})=x(t) \cdot e^{j 2 \pi f_{0} t}$

When reflected signals are added to a direct signal, the quality of the reception becomes worse. Multipath-propagation interference varies the signal intensity and produces intersymbol interference (ISI). These cases are modeled in the channel, which is defined as a Rice channel. In this type of channel, the Gaussian channel and its characteristics also be present. The received signal suffers a multipath channel with distinct waves. With the additive white Gaussian noise omitted, it can be expressed as:

$S^{\prime}(\mathrm{t})=\sum_{i=1}^{m} a_{i}(t) S\left(t-\tau_{i}(t)\right)$ which give the following equations:

$S^{\prime}(\mathrm{t})=\sum_{i=1}^{m} a_{i}(t) \cdot e^{-j 2 \pi f_{0} \tau_{i}(t)} \cdot x\left(t-\tau_{i}(t)\right) \cdot e^{j 2 \pi f_{0} t}$

where $a_{i}(\mathrm{t})=\beta_{i}(\mathrm{t}) \cdot e^{\alpha_{i}(\mathrm{t})}$ and $\alpha_{i}(\mathrm{t})$ designate the attenuation and the delay of the $\mathrm{i}$-th path. The received signal $S^{\prime}(\mathrm{t})$ can be rewritten as the baseband signal $y(t)$.

$y(\mathrm{t})=\sum_{i}^{m} c_{i}(t) x\left(t-\tau_{i}(t)\right)$

Where

$c_{i}(\mathrm{t})=a_{i}(t) e^{-j 2 \pi f_{0} \tau_{i}(t)}$

The Doppler shift affects the attenuation $a_{i}(t)$ periodically and is relatively small when compared with the carrier frequency $f_{0}$.

A simulation of the power requirements for the terrestrial DVB-T standard applied the following mathematical model to describe the channels with echoes. A certain number of echoes can be taken into consideration. In the Rician channel model, the Gaussian channel exists as well as its characteristics. The signal is completed with the reflection of signals in different ways. The received signals, $y(t)$ according to [28] becomes:

$$
y(t)=\frac{\rho_{0} x(t)+\sum_{i=1}^{m} \rho_{i} e^{-j 2 \pi \alpha_{i}} x\left(t-\tau_{i}\right)}{\sqrt{\sum_{i=0}^{m} \rho^{2}{ }_{i}}}
$$

The mathematical equation, which described the influence of Rayleigh channel on the signal:

$$
y(t)=\frac{\sum_{i=1}^{m} \rho_{i} e^{-j 2 \pi \alpha_{i}} x\left(t-\tau_{i}\right)}{\sqrt{\sum_{i=0}^{m} \rho^{2}{ }_{i}}}
$$

where $\rho_{0}$ is the attenuation in the line of sight of the transmitter, $\rho_{i}$ is the attenuation in echo path $i, \theta_{i}$ is the phase rotation in the echo part $i$, and $\tau_{i}$ is the relative delay time in the echo part. The Rice Factor $K$ designates the signal ratio by way of the line-of-sight broadcasting path to the sum in all echo ways (8).

$$
K=\frac{\rho_{0}}{\sum_{i=1}^{m} \rho^{2}}
$$




\section{B. MER Performance Analysis}

Intermodulation (IMD), $\mathrm{C} / \mathrm{N}$, and $\mathrm{RF}$ Level that can be used to evaluate the quality of service $(\mathrm{QoS})$ for DVB-H/T/T2 reception. The most useful one is the MER, which is used to quantify the performance of a digital radio (or digital TV) transmitter or receiver in communication, and it provides a clear and fast overview of the echoes and overlaps measurements; therefore, we highlight the MER.

In the OFDM system, a random bit sequence is generated, and then the bits are mapped into 64-QAM symbols. The $I$ and $Q$ values of this sequence are stored as the $\tilde{I}_{j}$ and $\tilde{Q}_{j}$ array. The $\tilde{I}_{j}$ and $\tilde{Q}_{j}$ array is next used to form the OFDM frequency domain signal, in which the resulting OFDM symbols $I_{j}, Q_{j}$ are mapped.

MER measurement of OFDM in SFN is used to measure the modulation quality [29], and practically its value is enough (according to measurements made on SFN) to judge the absence or the presence of the echoes in SFN.

MER over several symbols $\mathrm{N}$ (is the number of points in a measurement) is defined as:

$$
M E R=\frac{\sum_{j=1}^{N}\left(\tilde{I}_{j}^{2}+Q_{J}^{2}\right)}{\sum_{j=1}^{N}\left[\left(I_{j}-\tilde{I}_{j}\right)^{2}+\left(\mathrm{Q}_{j}-Q_{j}\right)^{2}\right]}
$$

where: $I_{j}, Q_{j}, \tilde{I}_{j}, \tilde{Q}_{j}$ are the ideal and quadrature components of the $\mathrm{j}$-th measured/ referenced OFDM signal, respectively.

\section{Intermodulation Performance Analysis}

IMD is the most useful signal analyzer measurements and widely used and is quite an appealing metric of linearity, which is very important to prevent it, for radiofrequency. In a Digital Terrestrial TV, IMD is a crucial measurement caused by nonlinearities effects in the DVB system. IMD measurement can be particularly troublesome for high-frequency amplifiers for radio communications; also can automatically detect the fundamental and third-order distortion that forms a principal limit to the circuit linearity. The introduction of third-order intermodulation is due to the non-linear characteristic.

\section{Problematic with the Proposed Remedy Solution}

\section{A. Problematic}

The GF is usually installed in high and isolated stations, the transmitting antenna designed to cover the shadow area optimally. The exemplary GF installation problem is the unavoidable RF coupling between the broadcast signal from the antenna patterns of broadcasting stations and the signal picked up by the receiving antenna.

If the coupling level is too high, this turns into a risky and challenging situation that can easily destruct the equipment hardware due to the positive return path. In the case where the GF is located within an SFN, The problem becomes even more complicated. To remedy this problem, we use a Digital Echo Canceller in the digital processing module of a GF.
In the SFN overlap area, the weaker of the received signals from multipath directions is deemed considered as an echo. Thanks to the global positioning system (GPS), the synchronization of the transmitters of the broadcasting centers allows echoes to be placed precisely at guard intervals (GI) which length depends on the duration of the echoes.

The problem worsens considerably, the COFDM solution, however, does not readily apply in domains where the user density is low, and impediments such as distance and terrain create challenging obstacles to conventional approaches to the DTTB network. The overview of DVB standards, namely, DVB-T/H/T2, was not able to solve the echoes squarely and overlaps phenomenon using COFDM techniques. From a physical point of view, the Overlap effects (between the antennas) are inevitable.

\section{B. Remedy}

For good isolation between the Tx and Rx antennas, the solution is a digital echo cancellation based on a software solution of an echo canceller GFs that can resolve the most challenging echo conditions. Therefore, to limit isolation between the Rx and Tx antennas and suppress echoes with interference in SFN, the PGF can optionally incorporate two echoes cancelling solutions DAE and DEEC.

DAE is a powerful and useful tool to remove echoes whose gain margin (GM) level can reach up -10 dB Moreover, especially in SFN operation, and in severe reception conditions, DAE can to remove multipath propagation impacts by equalizing the amplitude distortions of the GF received distorted signal spectrum that is created by very near echoes.

The DEEC is also able to suppress echoes but with considerable GM where the echo levels exceed the input signal by 24 $\mathrm{dB}$ and can often remove Doppler Effect. DEEC is integrated to avoid multipath, as well as Doppler echoes. The GF cancels feedback echoes with a GM up to $24 \mathrm{~dB}$, and three gaps echo, giving a selective cancellation that would go until $37,6 \mu \mathrm{s}$. Fig. 1 shows a correct example of the positioning of the RX and TX antennas.

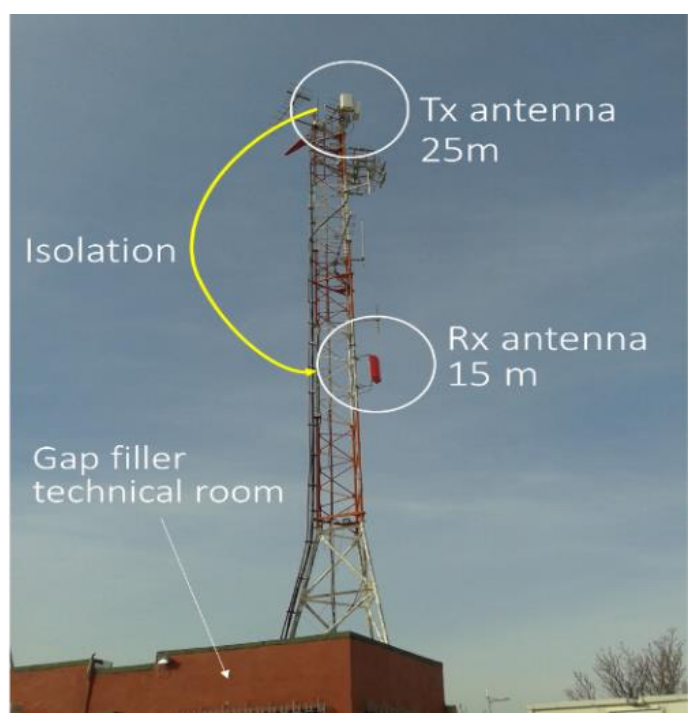

Fig. 1. Correct Positioning of the TX and RX Antennas Seeking Maximum Isolation. 
We select the $\mathrm{Rx}$ antenna position to at least 15 meters away from the TX antenna (if possible). We try to target the Tx and $\mathrm{Rx}$ antennas for opposite sides.

Fig. 2 shows a simplified system Topology containing a high-power transmitter and GF as well as the Field Test Receiver Measurements: Covered by GF interfere with the main transmitter at which the outdoor measurements are performed.

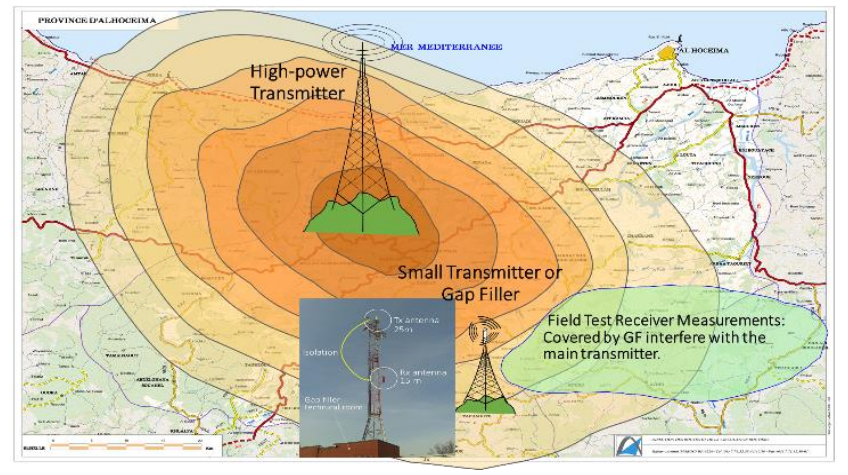

Fig. 2. Topology for SFN DVB-T/T2 Network Measurements.

\section{ECHO CANCELLER: FunCTIONAL DESCRIPTION}

Fig. 3 shows the block diagram representation of the digital GF internal composition. The system carries out a downconversion of the receiving RF signal to an intermediate frequency (IF), filters the resulting signal, and reconverts it back to RF that it is amplified before rebroadcasting starts.

When the echo signal level 10dB inferior to the principal signal, the standard GF can work without any echo canceller in SFN. In the case where reception conditions are particularly difficult in SFN, the need for DAE for an echo signal level up to $15 \mathrm{~dB}$ or DEEC for echo signal level higher than the fundamental signal is extremely important.

\section{A. DEEC: Technical Representation}

An adaptive LMS filter is the indispensable content of DEE that can detect the eventual feedback echoes and echoes of the SFN then can usually delete them all or part of the output. The DEEC high-performance gives it great robustness against echoes in the most challenging reception conditions. DEEC can remove high return echo levels, suppress echoes with greater GM, and cancel Doppler Effect improving MER performance:

Output MER is elevate to $27 \mathrm{~dB}$ for a $20 \mathrm{~dB}$ GM.

Output MER is superior to $24 \mathrm{~dB}$ for a $24 \mathrm{~dB}$ GM.

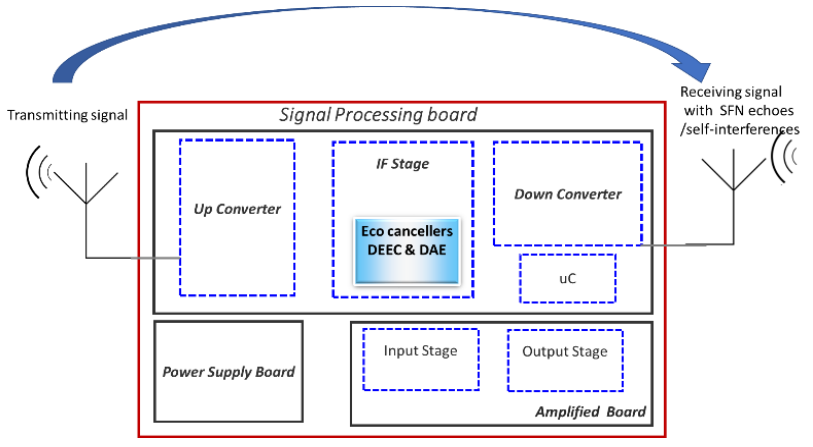

Fig. 3. Simplified Gap Filler Block Diagram.
The cancellation coverage depends on three temporal gaps, essentially $6 \mu$ s large each, appropriate in continuous mode, and they entirely fully include a range from $0,5 \mu$ s to $18,5 \mu \mathrm{s}$. The three gaps can move individually, and they can be extended to 37,6 $\mu$ s. The settings and measuring values of Fig. 4 and 5 are shown in Table I.

The DEEC graphs, of the input-output signal selection, are shown in Fig. 4 and 5, respectively.

DEEC can also suppress any multipath and even Doppler echo in a mobile cancellation gap going up to $37,6 \mu \mathrm{s}$. The echo cancellation level (20dB) with DEEC is presented in Fig. 4(a) and 4(b). The 20dB level is higher than the signal using DEEC and with the best output MER $(27 \mathrm{~dB})$.

The return echoes can reach high levels at the input signal. In that case, to obtain an active suppression of this echo of return, its corresponding cancels gap use an external reference. This cancellation gap system should always be active, which is shown in Fig. 6.

TABLE. I. SETting AND MEASURING THE VALUE OF Fig. 4 AND 5

\begin{tabular}{|l|l|l|}
\hline \multicolumn{1}{|c|}{ Parameters } & Fig. $\mathbf{~}$ & Fig. 5 \\
\hline TV/Radio Standard: & OFDM DVB-T/H & OFDM DVB-T/H \\
\hline Channel & 32 & 32 \\
\hline UHF RF & $562 \mathrm{Mhz}$ & $562 \mathrm{Mhz}$ \\
\hline Channel Bandwidth: & $8 \mathrm{Mhz}$ & $8 \mathrm{Mhz}$ \\
\hline Signal level & $-36.50 \mathrm{dBm}$ & $-6.50 \mathrm{dBm}$ \\
\hline Attenuation & $0 \mathrm{~dB}$ & $31 \mathrm{~dB}$ \\
\hline BER & $0.0 \mathrm{e}-8$ & $0.0 \mathrm{e}-8$ \\
\hline MER & $28.1 \mathrm{~dB}$ & $28.7 \mathrm{~dB}$ \\
\hline DEMOD & MPEG & MPEG \\
\hline
\end{tabular}

Siglv1 $=-36.50 \mathrm{dBm} \quad$ Att $=0 \mathrm{~dB} \quad$ Ch 32

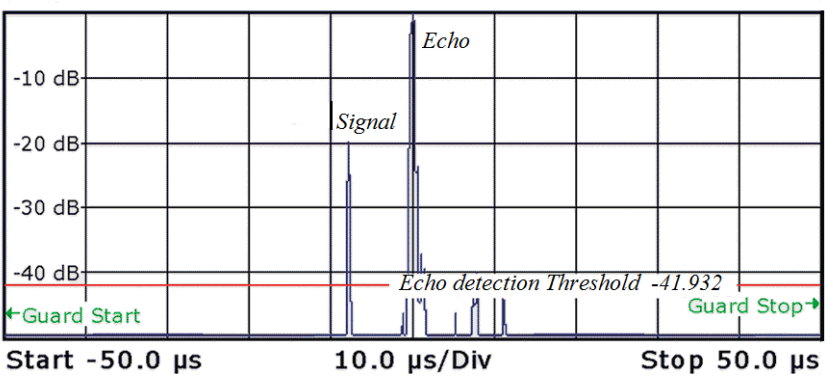

Fig. 4. DEEC Echo Canceller: Input Signal Spectrum.

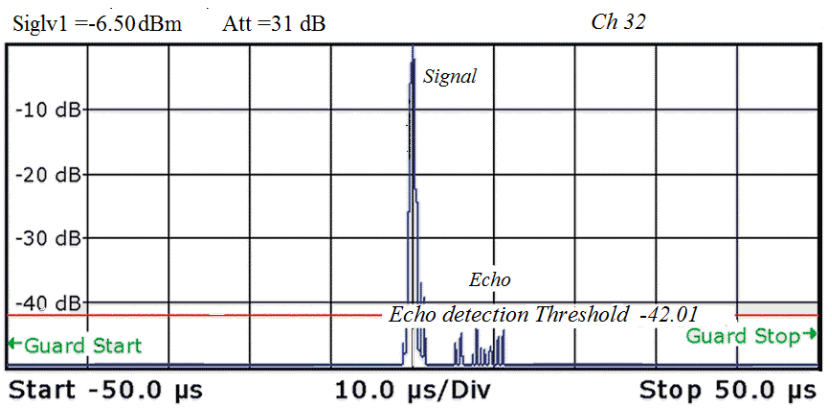

Fig. 5. DEEC Echo Canceller: Output Signal Spectrum. 


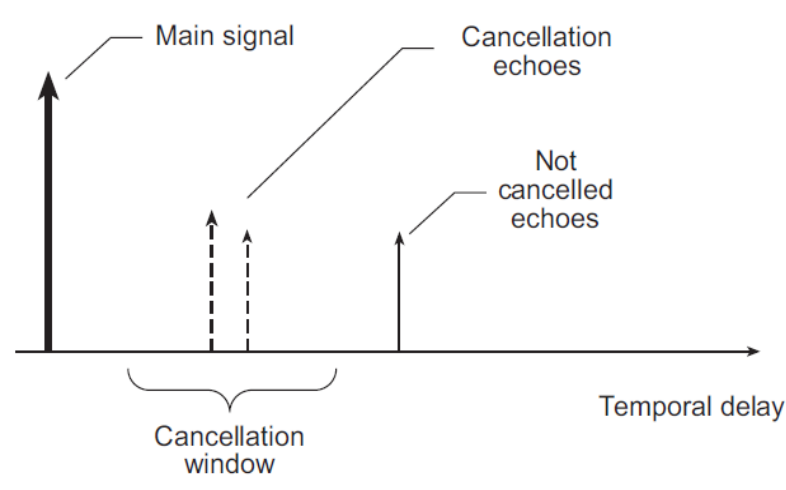

Fig. 6. Cancelation Gaps.

Fig. 7 shows the configurations of the most common DEEC gap positions, which cover a wide variety of cancellation scenarios.

There are three configurable cancellation gaps in the DEEC, namely:

Continuous cancellation: a continuous gap of $18 \mu \mathrm{s}$

Selective cancellation: up to $37,6 \mu \mathrm{s}$

Cancel any echo present in the cancellation gap (multipath propagation).

The following are the user-configurable parameters for the canceller:

Gap $N^{\circ} 1$ : Defines the cancellation velocity of gap $\mathrm{N}^{\circ} 1$.

Gap N $N^{\circ}$ : Defines the cancellation velocity of gap $\mathrm{N}^{\circ} 2$.

Gap No3: Defines the cancellation velocity of gap $\mathrm{N}^{\circ} 3$.

The cancellation velocity is directly related to the ability to track and cancel echoes with fast frequency and amplitude changes as present on Doppler, Rice, or Rayleigh channels.

However, increasing the speed of a cancellation gap creates a penalization on the output signal MER. The users should examine the different speeds to find a compromise between cancellation performance and output signal MER.

Gap $\mathrm{N}^{\circ} 1$ : Activates/deactivates the cancellation gap $\mathrm{N}^{\circ} 1$.

Gap $\mathrm{N}^{\circ}$ 3: Activates/deactivates the cancellation gap $\mathrm{N}^{\circ} 3$.

Gap $N^{o} 1$ and Gap $N^{\circ} 3$ are designed to suppress SFN echoes. These kinds of echoes may not appear/be relevant in every cancellation scenario, so these gaps can be switched off to improve the output signal MER, as explained above.

However, gap $\mathrm{N}^{\circ} 2$ is designed to challenge feedback echoes (which can be higher than the fundamental input signal), so it must continuously be activated to avoid feedback amplification that may harm and damage the equipment.

Gap N ${ }^{\circ}$ : Defines the cancellation starting point of the gap $\mathrm{N}^{\circ} 1$. The available values are $0.5 \mu \mathrm{s}-31.6 \mu \mathrm{s}$; the default value is $12.0 \mu \mathrm{s}$.

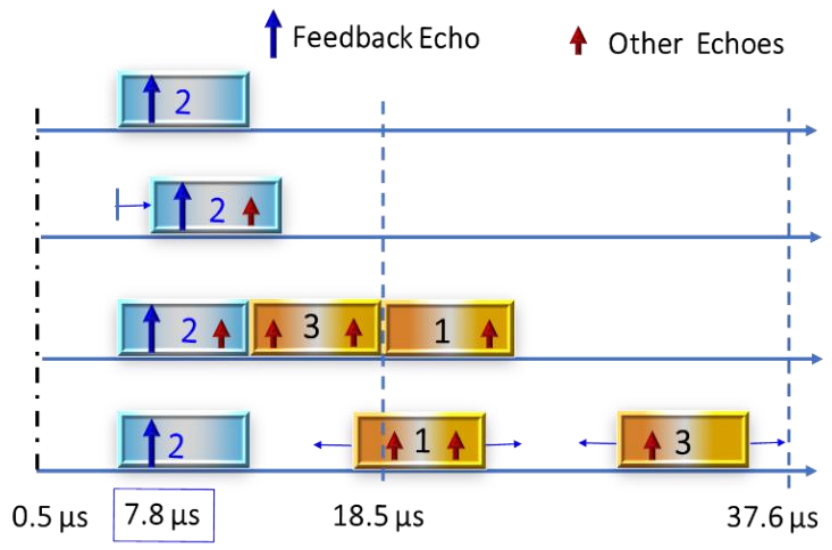

Fig. 7. Possible Cancellation.

Gap No2: Represents the cancellation starting point of the gap $\mathrm{N}^{\circ} 2$. The available values for setting up the gap $\mathrm{N}^{\circ} 2$ position are $5.0 \mu \mathrm{s}-36.1 \mu \mathrm{s}$; the default value is $6.0 \mu \mathrm{s}$.

Gap N $N^{\circ}$ : Defines the cancellation beginning point of the gap $\mathrm{N}^{\circ} 3$. The available values are $0.5 \mu \mathrm{s}-31.6 \mu \mathrm{s}$; the default value is $18.0 \mu \mathrm{s}$.

Each of these gaps has a time duration of almost $6 \mu \mathrm{s}$ in an $8 \mathrm{MHz}$ bandwidth operation.

\section{B. DAE: Technical Representation}

The DAE echo canceller is designed to effectively moderate echo conditions guaranteeing a GM level of support $15 \mathrm{~dB}$ $\mathrm{GM}$ and equalizing spectrum for robust feature extraction for the more robust fight against echoes. Furthermore, DEA is capable of removing any echo caused by the presence of coupling between the broadcasting, receiving antenna, more precisely, the input signal whose delay varies between zero and $8 \mu \mathrm{s}$. DAE circuit can also rectify the distortions in the GF input signal amplitude, which are within the cancellation gap, caused by multipath propagation. The settings and measuring values of Fig. 8 and 9 are shown in Table II.

The DAE graphs of the input-output signal selection are shown in Fig. 8 and 9, respectively.

TABLE. II. Setting AND MEASURING THE VALUE OF FIg. 8 AND 9

\begin{tabular}{|l|l|l|}
\hline Parameters & Fig. 8 & Fig. 9 \\
\hline TV/Radio Standard & OFDM DVB-T/H & OFDM DVB-T/H \\
\hline Ch & 32 & 32 \\
\hline UHF RF & $562 \mathrm{Mhz}$ & $562 \mathrm{Mhz}$ \\
\hline Channel Bandwidth: & $8 \mathrm{Mhz}$ & $8 \mathrm{Mhz}$ \\
\hline Signal level & $-40 \mathrm{dBm}$ & $16 \mathrm{dBm}$ \\
\hline Attenuation & $0 \mathrm{~dB}$ & $20 \mathrm{~dB}$ \\
\hline BER & $0.0 \mathrm{e}-8$ & $0.0 \mathrm{e}-8$ \\
\hline MER & $28.1 \mathrm{~dB}$ & $28.7 \mathrm{~dB}$ \\
\hline DEMOD & MPEG & MPEG \\
\hline
\end{tabular}




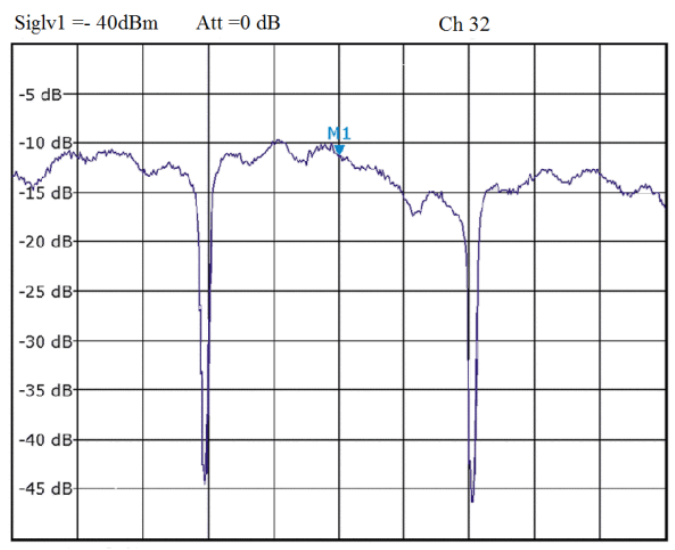

Fig. 8. Cancellation Example of an Echo up to $10 \mathrm{~dB}$ with DEA Echo Canceller. Input Signal Spectrum Equalization.

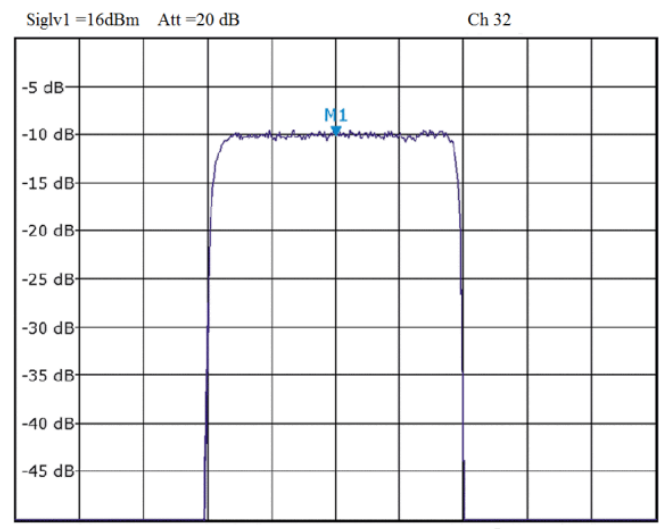

Fig. 9. Cancellation Example of an Echo up to $10 \mathrm{~dB}$ with DEA Echo Canceller. Output Signal Spectrum Equalization.

\section{RESUltS AND DisCUSSION}

\section{A. Measurement Results}

The experimental measurements have been conducted mainly to evaluate the MER performances of the PGF scheme with a DEEC \&DAE, compared with SGF, over a Rician multipath fading channel. The materials listed below are required during the installation of a GF, which are used in the results of indoor measurements.

- Computer with Ethernet cable.

- Signal analyzer ANRITSU model MS2712E or equivalent.

- Power Meter and Dummy load.

- RX antenna must have high directivity and high Ratio Front/Back.

- The professional Yagi antenna is suitable for this kind of installation.

The Tx antenna should not have a very open radiation diagram; ideally, the main lobe has $90^{\circ}$ to $180^{\circ}$. It is also essential to pay attention to the side lobes, as they can affect the $\mathrm{Rx}$ antenna and cause strong coupling (echo) between receiving and transmitting signals.
To perform the measurement equipment ROMAX TV Explorer HD + was used as a level meter. It is used to analyze the DVB-H/T/T2 signal providing several quality measurements (Power, C/N, MER, BER, constellation diagram, etc.). The results of the study could be useful for the DVB-T/H/T2 broadcast improvement, such as Tx adjustment and GF installation, to optimize the DTB efficiency.

Measurement points can be selected $1 \mathrm{Km}$ away from the transmitter location, which means that the measurement points that will possibly be on each radiation path should not be more than $20^{\circ}$.

\section{B. Intermodulation Measurements}

We consider passing the signal through a GF, which is measured with Signal analyzer ANRITSU. Fig. 8(a) and 8(b) show the IMD Shoulder performance measurements of frequency relative to the center frequency of $8 \mathrm{MHz}$ DVB-T versus Level measured, with $100 \mathrm{kHz}$ resolution bandwidth (RBW). Fig. 10 represents the Standard GF (SGF), without DEEC \&DAE, while Fig. 11 represents the PGF.

The nonlinear distortion in Fig. 10 caused by echoes phenomenon further brings an IMD Shoulder degradation performance of about $-16.08 \mathrm{~dB}$ compared to the result proposed method Fig. 11. Therefore, the proposed Echo Cancellers, incorporated in GF, can still be used to improve the IMD performance of the DTT system significantly.

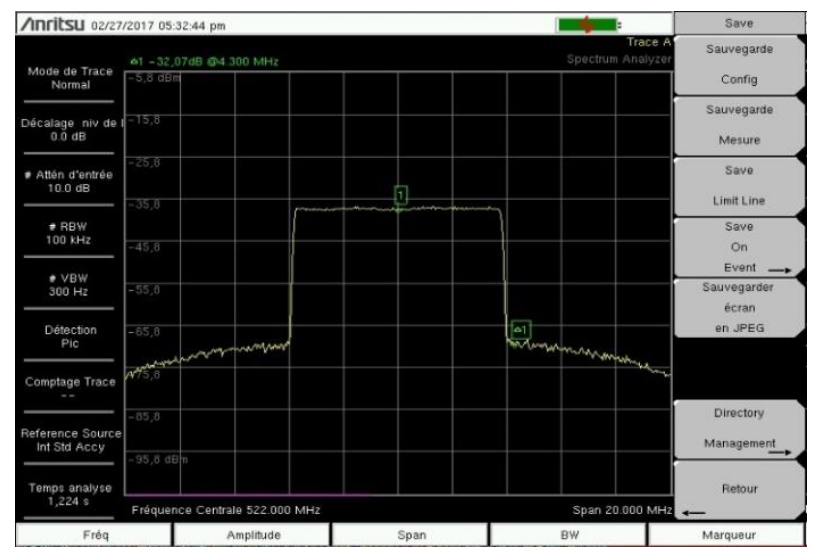

Fig. 10. IMD Measurement: $-5.8 \mathrm{dBm}$ Corresponds to the Average Output Power Test Interface of SGF.

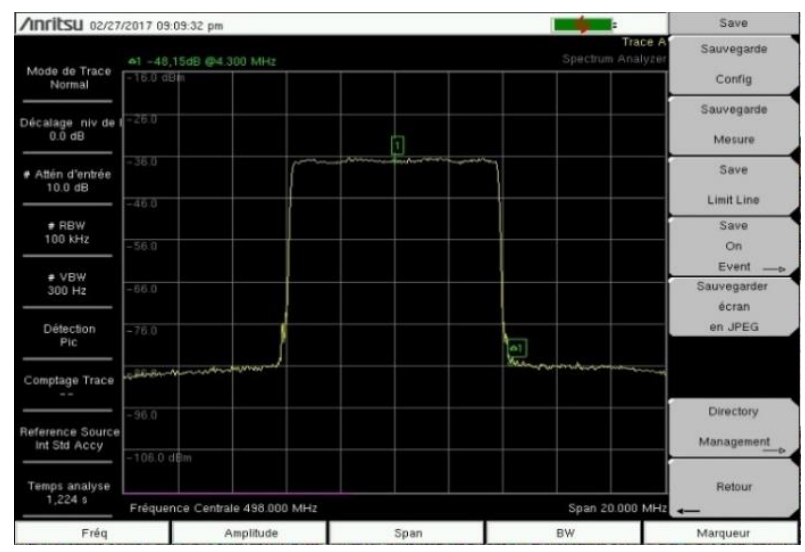

Fig. 11. IMD Measurement: PGF. 
It should be noted that, based on experience, the increasing attenuation can surely minimize the IMD contribution of the $\mathrm{RF}$ signal analyzer. The measure of the third-order distortion with a significant attenuation is not detectable because it corners with the measurement values located outside tolerance.

\section{MER Measurements}

Fig. 12 and 13 present MER/Constellation measurement of the signal received from the test interface of the SGF and PGF, respectively, giving their performance comparison, $0 \mathrm{~dB}$ corresponds to average output power. Fig. 12 shows the proven MER measurement for a DVB-T signal that was mainly affected by unwanted modulator phase noise. It shows that the transmission quality of DVB-T is not satisfactory.

Fig. 13 illustrates the PGF result, and the constellation diagram also depicts a high-quality DVB-T signal (the points are not scattered).

From Fig. 12 and 13, it can be observed that the MER performance of the PGF is better than SGF; an improvement can reach up to $15 \mathrm{~dB}$. We also observe that the DEEC \& DAE can improve the robustness of the constellation, free of noise and interferences that lead to the better average. Therefore, a higher MER value promotes wide broad coverage area

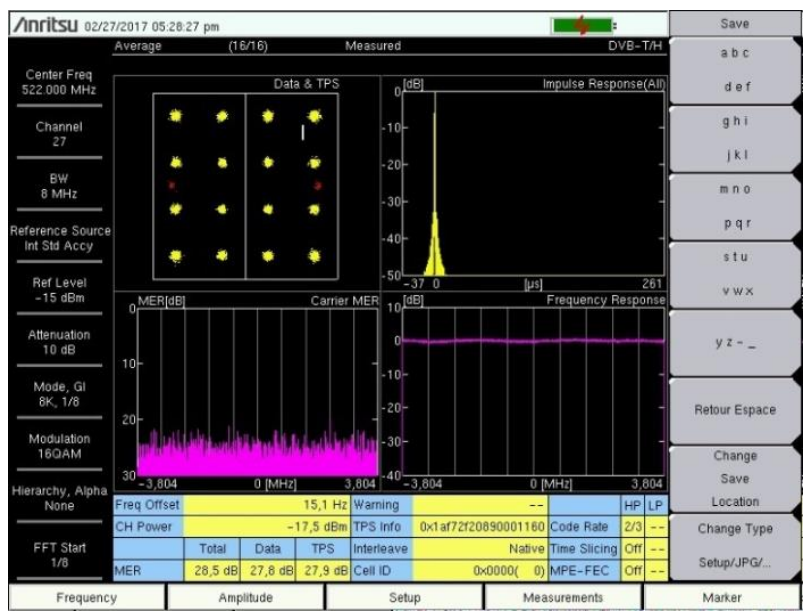

Fig. 12. MER and Constellation Measurement: SGF.

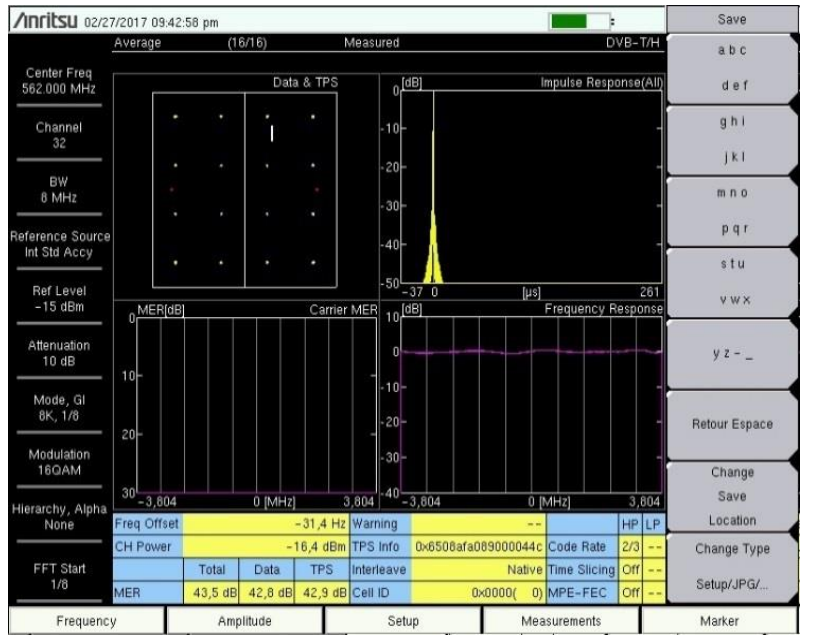

Fig. 13. MER and Constellation Measurement: PGF.

\section{Comparison between the PGF and the Adaptive GF Technique}

To further evaluate the performance of the proposed method, we compare it to another Echo Canceller method, more particularly, the adaptive GF technique. Fig. 14 and 15 ("MER vs. CARRIER") show the Echo Pattern of AGF technique compared to the PGF.

The average MER value is represented, in the screen, by a red line ted, which is measured in the whole channel (RMS) of about $37.4 \mathrm{~dB}$ (Adaptive GF) and $42.21 \mathrm{~dB}$ (PGF). It observed that the MER of PGF is better than the MER of AGF.

\section{E. Comparison of an Existing Technique}

Fig. 16 and 17 show the comparison simulation results (MER and receive signal levels) of different GF techniques obtained in four echo cancellation, namely standard GF(SGF), finite impulse response (FIR) filter, GF, adaptive GF, and the PGF.

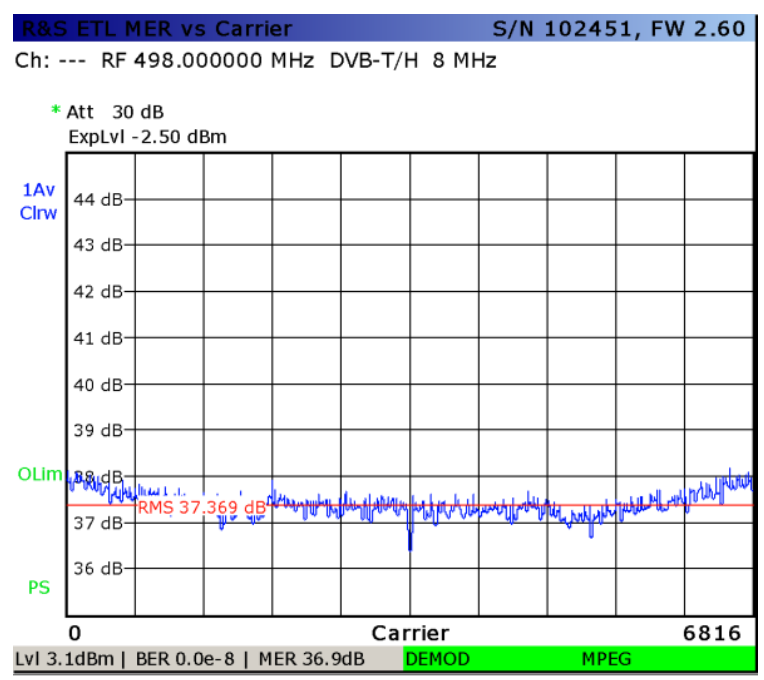

Date: 9.MAR.2016 15:06:36

Fig. 14. MER vs. Carrier of Adaptive GF.

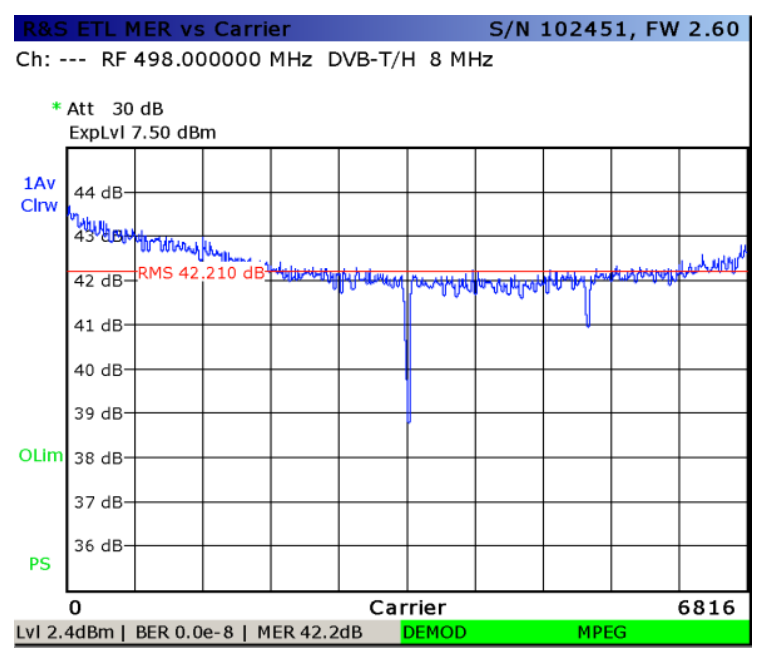

Date: 10.MAR.2016 11:59:29

Fig. 15. MER vs. Carrier of PGF. 


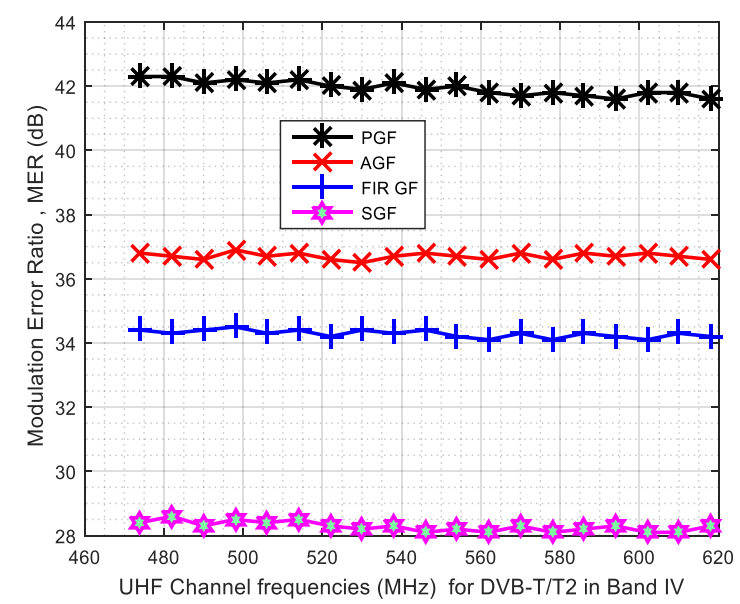

Fig. 16. MER vs. UHF Channel Frequencies Mhz.

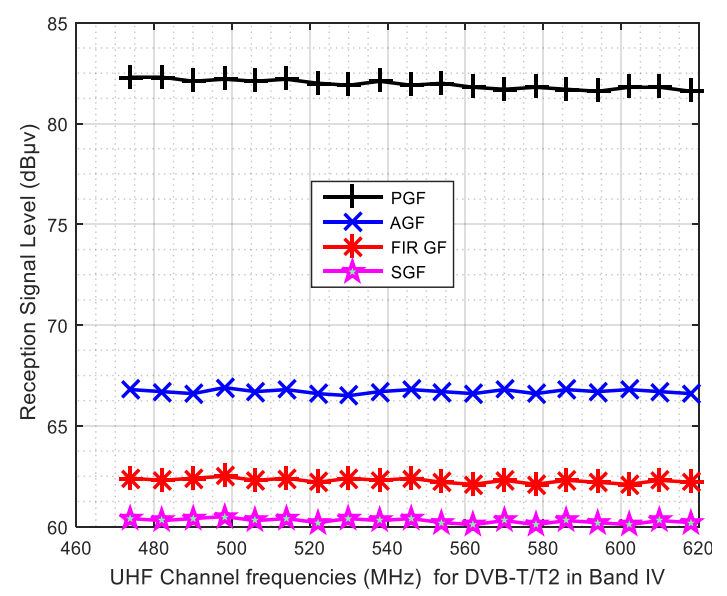

Fig. 17. Reception signal level vs. UHF channel frequencies Mhz.

Simulation results (Fig. 16 and 17) show perfect agreement with those obtained by measurements. Moreover, the MER and receive signal levels quality obtained by the PGF technique is much better than that obtained by SGF, FIR filter GF, and AGF.

\section{F. General Analysis and Discussion}

Table III summarizes the comparison between the different existing techniques obtained by four different Echo Cancellation techniques.

The Summary of analysis results, in Table III, illustrates that in four cases, the PGF outperforms the existing techniques. The proposed GF outperform standard GF(SGF), finite impulse response (FIR) filter (FIR GF)), GF and adaptive GF (AGF) techniques by $33 \%, 17 \%$, and $13 \%$, respectively, this outperformance is also characterized by:

Maximizing received signal quality: better than-60dBm level, MER.

Better than $37 \mathrm{~dB}$ and lower incidence of multipath.

Less coupling between the TX and RX antennas: Echo Level always less than $-20 \mathrm{~dB}$.

Can suppress high feedback echo levels.
TABLE. III. COMPARISON OF THE PROPOSED GF WITH OTHER GF TECHNIQUE

\begin{tabular}{|l|l|l|l|l|}
\hline $\begin{array}{l}\text { Echo cancella- } \\
\text { tion Techniques }\end{array}$ & MER dB & IND dB & $\begin{array}{l}\text { Echo } \\
\text { levels dB }\end{array}$ & $\begin{array}{l}\text { Signal quality } \\
\text { dBm }\end{array}$ \\
\hline SGF & 28.5 & -32.07 & -10 & -80 \\
\hline FIR GF & 34.5 & -37.8 & -16 & -71 \\
\hline AGF & 36.9 & -40.8 & -18 & -66 \\
\hline PGF & 42.2 & -48.15 & -22 & -58 \\
\hline
\end{tabular}

DEEC can also suppress any multipath, and even Doppler echo in a free cancellation gap going up to $37.6 \mu \mathrm{s}$.

Also, in light of the findings, and achievement of the aims and objectives of the study, the results (with directive antennas on both the gap filler side and the principal transmitter side) are depicted in Fig. 16 and 17, in terms of MER/Reception signal level vs. UHF channel frequencies $\mathrm{MHz}$ respectively, between transmitter and receiver. It can be noted that the proposed solution provides the most extensive MER, and therefore seems to be the most suitable to provide coverage in SFN with a reduced number of GF.

The result is mainly related to the highly efficient air interface of the conventional GFs and, in particular, to its advanced coding technique. What is not taken into account in this study is the effect of severe multipath on the modulation schemes (ideal synchronization is assumed).

The overall research approach would suffer more than the many competitors, which are based on OFDM modulation, more robust to multipath. Such a drawback could be partially compensated by the antenna directivity, which provides a kind of spatial filtering of the echoes. Among the OFDM-based systems, the most extensive MER is provided by the DVB-H solution. The selection of the research method suffers for the weak coding scheme on the physical layer (i.e., errorcorrecting codes) in SFN, which requires precise synchronization and more considerable bandwidth.

\section{CONCLUSIONS}

In this paper, the authors propose an efficient GF, including two echo cancellations, DEEC, and DAE, as a critical technique to solve the echo and interference problem in the SFN environment. Furthermore, it presents DAE and DEEC for ideally limit the isolation, in SFN, between the broadcasting and receiving antennas. The modified GF scheme (PGF) with DAE and DEEC echo canceller is proposed to cover shadow zones cancelling echoes with no self-interference from adjacent transmitters for DTT in SFN. Also, these GFs able to retransmit the RF signal under the most challenging echo and multipath propagation conditions removing high feedback echo levels. The PGF gives an excellent MER and can even remove the Doppler Effect. In our future work, we will use these areas as starting points for strengthening information protection for the new DVB generation in SFN.

\section{ACKNOWLEDGMENT}

The authors would like to acknowledge the moral and financial efforts of Dr. Wafaa Roky. 


\section{REFERENCES}

[1] H. Hamazumi, K. Imamura, N. Iai, K. Shibuya, and M. Sasaki, "A study of a loop interference canceller for the relay stations in an SFN for digital terrestrial broadcasting," IEEE. Global Telecommunications Conference (GLOBECOM'00) San Francisco, USA, pp. 167-171.2000

[2] M. Lanza, A.L. Gutiérrez, I. Barriuso, J.R. Pérez, M. Domingo, L. Valle, J. Basterrechea, J. Morgade, and P. Angueira , "Coverage optimization in single frequency networks using simulated annealing," IEEE International Symposium on Antennas and Propagation (APSURSI), Spokane, USA, pp. 2789- 2792.2011.

[3] A. Mattsson, "Single-frequency networks in DTV," IEEE Transactions on Broadcasting, vol. 51, DOI: 10.1109/TBC.2005.858419 ,no.4, pp. 413-422, Dec, 2005.

[4] S.S. Das; F.H.P. Fitzek; E.D. Carvalho; R. Prasad, "Variabl guard Interval OFDM in presence of carrier frequency offset," IEEE Global Telecommunication Conference(GLOBECOM), MO, USA, DOI: 10.1109/GLOCOM.2005.1578296,pp. 2937-2941. Dec.2005

[5] Hsiao-Chun Wu, "Analysis and characterization of intercarrier and interblock interferences for wireless mobile OFDM systems," IEEE Transactions on Broadcasting, vol. 52, DOI: 10.1109/TBC.2006.872989, no. 2, pp. 203 -210. June. 2006.

[6] Vincent Savaux, Moise Djoko-Kouam and Alexandre Skrzypczak, "Study of cyclic delay diversity for single frequency network using DRM Standard," European Wireless19th European Wireless Conference, Guildford, UK, pp. 1-6. April .2013

[7] Marta Lanza,; Ángel L. Gutiérrez, Jesús R. Pérez, Javier Morgade Marta Domingo, Luis Valle, Pablo Angueira and José Basterrechea, "Coverage optimization and power reduction in SFN using simulated annealing," IEEE Transactions on Broadcasting, vol. 60 DOI: 10.1109/TBC.2014.2333131,no. 3, pp. 474-485.Sep.2014

[8] El Miloud Ar-Reyouchi and Kamal Ghoumid,"Technical accuracy based on efficiency algorithm for measuring standing wave ratio in wireless sensor network," International Journal on Communications Antenna and Propagation vol. 9, n. 2, pp.137-14, April 2019.

[9] J.-D. Kim, Y.-S. Byun," A new inter-carrier interference cancellation using CP-ICA scheme in OFDM Systems," IEEE 65th Vehicular Technology Conference - VTC2007-Spring, Dublin, Ireland, DOI:10.1109/vetecs.2007.489, pp. 2369-2373. April .2007

[10] El Miloud Ar Reyouchi, Kamal Ghoumid, Amezian Koutaiba, Otman Mrabet, "MIMO-OFDM coded for digital terrestrial television broadcasting systems," World Academy of Science, Engineering and Technology 76, p. 617-621. Avril, 2013.

[11] El Miloud Ar Reyouchi, K. Ghoumid, K.Amezian, and O.Mrabet, "The powerful Alamouti code in MIMO-OFDM improvement for the next generation of terrestrial television broadcasting systems," International Journal of Engineering \& Technology IJET-IJENS. vol. 14, no. 1, pp. 33-42, January 2014.

[12] Ar-Reyouchi, El Miloud. " Optimisation des performances des réseaux de communications sans fil: Performances des réseaux de communications sans fil pour la télégestion des stations de la Télédiffusion TV/FM', https://www.amazon.com/Optimisationperformances-réseaux-communications-sans/dp/3841642861,Isbn: 978 3-8416-4286-8. Publisher: Paf ,June 2017.

[13] A. Gohn and J. Kim, "Implementation of LMS adaptive filter algorithm based on FPGA," 2019 IEEE 62nd International Midwest Symposium on Circuits and Systems (MWSCAS), Dallas, TX, USA, pp. 207 210,2019 .
[14] Mahmood Farhan Mosleh, Aseel Hameed AL-Nakkash, "Combination of LMS and RLS adaptive equalizer for selective fading channel," European Journal of Scientific Research, vol.43.no.1, pp.127-137, Jun 2010 ,

[15] R. Meier, E. DeMan, T. G. Noll, U. Loibl and H. Klar, “A 2 pm CMOS digital adaptive equalizer chip for QAM digital radio modems," IEEE Journal of Solid-State Circuits, vol.23, DOI: 10.1109/4.5946,No.5,pp. 1212-1217, November.,1988.

[16] X. Hou, and C. Yang, "Feedback overhead analysis for base station cooperative transmission," IEEE Transactions on Wireless Communications, vol. 15, DOI: 10.1109/TWC.2013.013013.120534, no. 7, July 2016.

[17] Ar-Reyouchi El Miloud, Lichioui, Rattal Salma, "A group cooperative coding model for dense wireless networks," International Journal of Advanced Computer Science and Applications. 10. 10.14569/IJACSA.2019.0100750, 2019

[18] H. Bawab, P. Mary, J. Hlard, Y. Nasser, and O. Bazzi, "Spectral overlap optimization for DVB-T2 and LTE Coexistence," IEEE Transactions on Broadcasting, vol. 64, no. 1, pp. 70-84, Mar. 2018.

[19] K. M. Nasr, J. P. Cosmas, M. Bard, and J. Gledhill, "Performance of an echo canceller and channel estimator for on-channel repeaters in DVBT/H Networks," IEEE Transactions on Broadcasting, vol. 53, DOI: 10.1109/TBC.2007.903612, no. 3, pp. 609-618, September 2007.

[20] F. Lindqvist and A. Fertner, "Frequency domain echo canceller for DMT-based systems," IEEE Signal Processing Letters, vol. 18, DOI: 10.1109/LSP.2011.2170679, no.12, pp. 713-716, .December.2011.

[21] P. P. Kadam, Z. Saquib and A. Lahane, "Adaptive echo cancellation in VoIP network," 2016 IEEE International Conference on Engineering and Technology (ICETECH), Coimbatore, pp. 295-299,2016.

[22] C. Rocha, C. Akamine, G. Bedicks, E. L. Horta and G. Stolfi, "Adaptive gap filler for digital terrestrial television," IEEE International Symposium on Broadband Multimedia Systems and Broadcasting, Beijing, China, DOI: 10.1109/BMSB.2014.6873535 pp.1-6. June.2014.

[23] Michael Mao Wang, "Dynamic gain management for on-channel repeaters," IEEE Transactions on Broadcasting, vol.59, DOI: 10.1109/TBC.2013.2284417, no.4, pp. 685-692. Dec.2013

[24] M. M. Sondhi, "The history of echo cancellation", IEEE Signal Processing Magazine, vol. 23, DOI: 10.1109/MSP.2006.1708416, no. 5, pp. 95 -98. September .2006

[25] Fuyun Ling, "Achievable performance and limiting factors of echo cancellation in wireless communications," Information Theory and Applications Workshop (ITA), San Diego, USA, DOI: 10.1109/ITA.2014.6804210, pp.1-8.Feb.2014.

[26] Bundit Ruckveratham , Sathaporn Promwong'Empirical single frequency network threshold for DVB-T2 based on laboratory experiments" Turkish Journal of Electrical Engineering \& Computer Sciences, pp: $3342-3355,2019$

[27] Ruckveratham B, Promwong S. "Evaluation of SFN gain for DVB-T2. In: International Conference on Digital Arts, Media and Technology", Chiang Mai, Thailand. New York, NY, USA: IEEE. pp. 85-88,2017.

[28] ETSI EN 300744 V1.4.1 (2001-01). European Standard. Digital Video Broadcasting (DVB); Framing structure, channel coding and modulation for DTT. ETSI, 2001.

[29] Yin Wang, Zhaowu Chen and Ke Gong, "MER performance analysis of M-QAM OFDM with wiener phase noise," IEEE International Conference on Microwave and Millimeter Wave Technology, DOI: 10.1109/ICMMT.2007.381517 pp. 18-21, April 2007. 